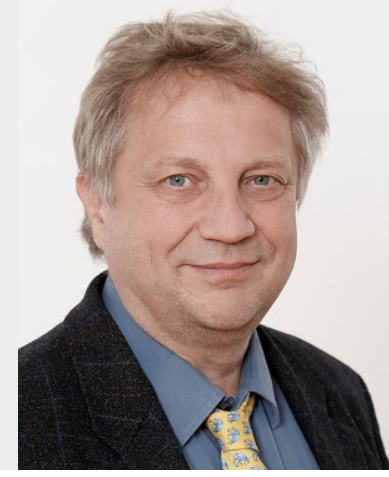

॥ Psychotherapie kann Plexusanästhesie aber nicht Körperakupunktur integrieren

\author{
Dr. Jürgen Bachmann
}

Schmerzmedizin - Orthopädie - Translationale Medizin

Augustastrasse 25c, 45525 Hattingen, Deutschland

dza@dr-bachmann-hattingen.de

\title{
Das Große im Kleinen
}

Liebe LeserInnen und KollegInnen,

vor Ihnen liegt die erste Ausgabe 2021: Wir starten ins Jahr 1 nach Corona. Die Notwendigkeit einer Rubrik mit News rund um die einschlägigen Möglichkeiten der chinesischen Medizin und integrative Ansätze im Management der COVID-19-Infektion haben sich leider noch nicht erübrigt. Aus internationaler Perspektive wird die aktuelle Diskussion immer differenzierter: Staatliche Maßnahmen im Management der Pandemie, die $\mathrm{Zu}$ lassung von Impfstoffen und Therapeutika, deren Entwicklung Vermarktung und Vertrieb sind hierzu nur einige Schlagworte und ich verweise sie gerne auf die wieder einmal facettenreiche Aufbereitung von Axel Wiebrecht.

Ich freue mich, dass im wissenschaftlichen Teil unseres Heftes nicht nur „Wissenschaft für die Kitteltasche“ diesmal mit dem Thema Kopfschmerzen bei Kindern von C. Chiu, sondern auch eine Originalarbeit von Kollegen P. Panhofer et al. präsentiert wird, in der die Patientensicht als wesentlicher Eckpfeiler evidenzbasierter Medizin in den Fokus genommen wird: Das Vorliegen von Schlafstörungen verändert die Patientenpräferenz gegenüber einer integrativen Behandlung von Übergewicht und Adipositas.

Im Journal Club thematisiert H. Haller eine Arbeit zur methodischen Qualität von Akupunkturstudien in den letzten 50 Jahren und $C$. Colling beschreitet Neuland und widmet sich nicht einem einzelnen Fachartikel, sondern dem wissenschaftlichen Diskussionsverlauf anlässlich eines Artikels zur Methode der Emotional Freedom Techniques.

Der Schwerpunkt ist der Ohrakupunktur gewidmet: T. Ots und A. Rubach stellen neben J. Seeber und J. Gleditsch ihren eigenen pragmatischen Ansatz dar und liefern uns als Rubrikenverantwortliche ein breites Spektrum von Vergessenem und Neuem: M. Hammes unterstreicht, dass die Integration der Ohrakupunktur von West nach Ost erfolgte, T. Ots zeichnet die okzidentale Historie der Aurikulomedizin nach. Unterschiedliche Nachweisverfahren zur Punktsuche münden in unterschiedliche Somatotopien, G. Riehl, F. Bahr und P. Macek zeigen ihre Schlussfolgerungen auf. Last, not least schöpfen die Beträge von $H$. J. Weise, M. Bijak und nochmal G. Riehl aus der praktischen Anwendung.

Deutsche Zeitschrift für Akupunktur 2021 • 64 (1): 1-2

https://doi.org/10.1007/s42212-021-00362-z

(c) Springer Medizin Verlag GmbH, ein Teil von Springer Nature 2021
Auch unsere „Kolumne“ von Frau Kollegin Tauber-Bachmann ist dem Thema gewidmet!

Einmal mehr möchte ich hier Aufmerksamkeit auf die Bedeutung der Rahmenbedingungen unserer Tätigkeit als Akupunkteure lenken: Sie ist nicht unabhängig von Berufs- und Gesundheitspolitik! Und die ist immer wieder gut für konflikthafte Entwicklungen und administrative Merkwürdigkeiten.

Nicht alle konflikthaften Entwicklungen führen unmittelbar in eine bessere Zukunft. Die Darstellung von D. Irnich zu den Hintergründen der Konflikte in der Hufelandgesellschaft lässt uns die Vorgänge aber besser verstehen. Es bedarf nun einer optimistischen Veranlagung, hieraus Hoffnung auf eine Stärkung der Akupunktur im Rahmen integrativer Ansätze zu schöpfen: Akupunkteure und andere energetisch arbeitende Therapeuten bringen nur sich und ihre Zeit in die Therapie ein, mithin eine Ressource, die weder ohne Weiteres skalierbar ist noch in wesentlichem Umfang beiläufige Umsätze aus Produkten, Pharmazeutika oder Gerätschaften generiert.

$\mathrm{Zu}$ den administrativen Merkwürdigkeiten: Die spezielle Qualifikation in der Akupunktur hat Eingang in den Kanon der seitens der Ärztekammer gelisteten und damit ankündigungsfähigen Zusatzqualifikationen gefunden, die der weit überwiegenden Zahl fachärztlicher Qualifikationen - nämlich derer mit klinischem Bezug - offenstehen. Umso unverständlicher ist es, wenn in Deutschland für die gesetzlich Versicherten im Rahmen der Abrechnungsvorgaben des einheitlichen Bewertungsmaßstabes (EBM) zwar ein Teil der fachärztlichen Kapitel (z. B. 22 Psychosomatische Medizin und Psychotherapie und 16 Neurologie und Neurochirurgie) in der Präambel das Kapitel schmerztherapeutische Verfahren (Kapitel 30.7) insgesamt als abrechenbar verzeichnen, für einen anderen Teil, z. B. die Nervenärzte und Psychotherapeuten (Kapitel 21 und 23), die Akupunktur (Kapitel 30.7.3) im Gegensatz zu den Kapiteln 30.7.1 und 30.7.2 (Schmerztherapeutische Versorgung bzw. Behandlungen) jedoch nicht ausweisen. Im Ergebnis kann also eine integrative Behandlung eine psychotherapeutische Behandlung mit einer Plexusanästhesie verknüpfen, nicht jedoch mit einer Körperakupunktur.

Eine kurzfristig anberaumte Umfrage unter Akupunktureminenzen mit psychotherapeutischem Arbeitsschwerpunkt galt der Frage, ob denn fachlich begründete Zweifel an einem positiven Zusammenwirken psychotherapeutischer und funktionell-somatischer Verfahren wie der Akupunktur benennbar seien. Ergebnis: keine Zweifel, nada, njet, niente. Die administrative Merk- 
würdigkeit bleibt auf fachlicher Ebene unerklärlich und wird damit obsolet.

\section{》) Das Kapitel Ohrakupunktur kommt im EBM gar nicht erst vor}

Das Kapitel Ohrakupunktur kommt im EBM gar nicht erst vor. Unter den Denklimitationen der Versorgung im GKV-System könnten Sie sich die Lektüre unseres Schwerpunktes also sparen - aber ich bin überzeugt, dass Sie sich auf Höhe der Zeit befinden und dieser Anmutung längst nicht mehr unterliegen.

In diesem Sinne wünsche ich Ihnen eine erhellende Lektüre!

Ihr

Jürgen Bachmann

Interessenkonflikt. J. Bachmann gibt an, dass kein Interessenkonflikt besteht.

\section{Das Dilemma mit den Meta-Analysen am Beispiel Rückenschmerz}

Stellungnahme des Wissenschaftszentrums der Deutschen Ärztegesellschaft für Akupunktur

Im Dezember letzten Jahres wurde von Mu et al. eine aktualisierte Cochrane-Meta-Analyse der Studienlagen zum Nutzen der Akupunktur bei chronischen Rückenschmerzen publiziert [2]. Die Autoren analysieren 33 Studien mit 8270 Patienten. Nadelinterventionen mit Akupunktur wird im Vergleich zu keiner Intervention eine bessere Wirksamkeit in der unmittelbaren Schmerzreduktion und Funktionsverbesserung zugeschrieben. Im Vergleich zu Standardschmerzbehandlungen verbessert eine Nadelakupunktur insbesondere die körperliche Funktion und die physische Komponente der Lebensqualität. Die Autoren können zudem einen statistischen Unterschied zu Sham-Akupunkturinterventionen feststellen. Die klinische Bedeutsamkeit wird jedoch als gering beurteilt. Die Autoren geben die Sicherheit ihrer Evidenzeinschätzung mit moderat bis sehr niedrig an, dies auch in Folge eines vermeintlichen hohen BiasRisikos der eingeschlossenen Studien.

Die online-publizierte Stellungnahme des Wissenschaftszentrum [3] widmet sich insbesondere dieser Lesart, wie sie auch in einer Newsmeldung des Deutschen Ärzteblattes [1] aufgegriffen wurde. Dabei werden von den Autoren die Fragen nach der Verblindung in Akupunkturstudien -also dem Vergleich zu Schein-Akupunktur-, der Zahl der Studienabbrecher in der Nachbeobachtung nach einem Jahr, aber auch einer vermeintlich ungenügenden klinischen Relevanz, über die Maßen als Negativkriterien angelegt. Dies kontrastiert vor dem Hintergrund weiterer Cochrane-Meta-Analysen zum Thema Rückenschmerz, aber auch anderen themenverwandten Analysen, in welchen ein anderer Maßstab angelegt wurde.

Dass die Akupunktur gegenüber einer Nicht-Behandlung einen relevanten klinischen Wirksamkeitsvorteil erzielt, und somit chronischen Schmerzpatienten effektiv zu helfen vermag, wird von den Autoren nicht entsprechend dargestellt.

\section{Literatur}

1. Hil/Aerzteblatt.De (2021) Was Akupunktur bei chronischen Rückenschmerzen bringt. Link: https://www.aerzteblatt.de/nachrichten/120467/Was-Akupunktur-beichronischen-Rueckenschmerzen-bringt (letzter Zugriff 19. Februar 2021) 2. Mu J, Furlan AD, Lam WY et al. (2020) Acupuncture for chronic nonspecific low back pain. The Cochrane database of systematic reviews 12:Cd013814

3. Fleckenstein J, Banzer W. (2021) Das Dilemma mit den Meta-Analysen am Beispiel Rückenschmerz - Stellungnahme des Wissenschaftszentrums der Deutschen Ärztegesellschaft für Akupunktur. Deutsche Zeitschrift für Akupunktur. (epub ahead of print)

Zugriff über: https://dx.doi.org/10.1007/s42212-021-00363-y

Johannes Fleckenstein und Winfried Banzer 